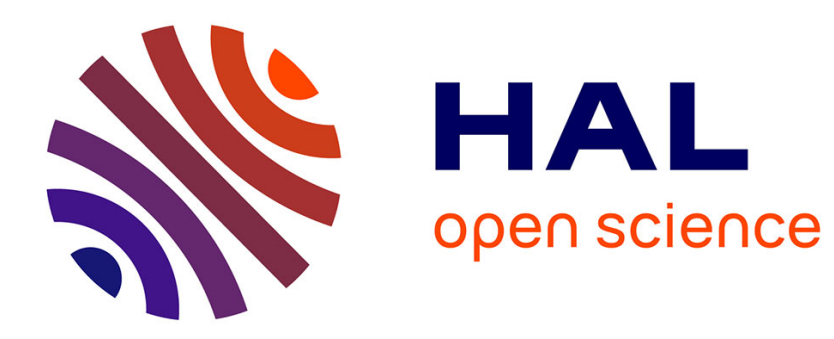

\title{
The variational structure of a nonlinear theory for spatial lattices
}

David Steigmann

\section{To cite this version:}

David Steigmann. The variational structure of a nonlinear theory for spatial lattices. Meccanica, 1996, 31 (4), pp.441-455. hal-00782160

\section{HAL Id: hal-00782160 \\ https://hal.science/hal-00782160}

Submitted on 29 Jan 2013

HAL is a multi-disciplinary open access archive for the deposit and dissemination of scientific research documents, whether they are published or not. The documents may come from teaching and research institutions in France or abroad, or from public or private research centers.
L'archive ouverte pluridisciplinaire HAL, est destinée au dépôt et à la diffusion de documents scientifiques de niveau recherche, publiés ou non, émanant des établissements d'enseignement et de recherche français ou étrangers, des laboratoires publics ou privés. 


\title{
The Variational Structure of a Nonlinear Theory for Spatial Lattices
}

\author{
D.J. STEIGMANN \\ University of Alberta, Department of Mechanical Engineering, 4-9 Mechanical Engineering Building, Edmonton, \\ Alberta, Canada, T6G $2 G 8$
}

(Received: 10 January 1995; accepted in revised form: 20 October 1995)

\begin{abstract}
A theory for spatial lattices is presented in a variational setting and conditions restricting stable deformations are discussed. In particular, new results on the second variation of the energy are established and used to generate pointwise necessary conditions for locally energy-minimizing configurations.
\end{abstract}

Sommario. Viene presentata una teoria per i reticoli spaziali in un ambito variazionale e sono inoltre discusse condizioni che limitano deformazioni stabili. In particolare vengono stabiliti nuovi risultati sulla variazione seconda dell'energia. Tali risultati vengono usati per stabilire condizioni necessarie puntuali per configurazioni che minimizzano l'energia localmente.

Key words: Structural lattices, Rods, Mechanics of solids

\section{Introduction}

In this work we examine some aspects of a nonlinear variational theory for three-dimensional deformations of extensible spatial rods. The theory that we consider is based on the notion of a one-dimensional continuum endowed with a kinematical and constitutive structure sufficient to represent the dominant features of the mechanics of suitably loaded thin three-dimensional elastic bodies. This theory belongs to a general class that has received considerable attention. An extensive account of such theories and their mathematical structure may be found in Antman's book [1]. The special theory considered here requires relatively modest empirical input with regard to constitutive equations, and incorporates a reasonably broad range of response, ranging from flexible cables to rods with significant flexural and torsional rigidity. It is thus well suited to applications.

Our contribution to this subject is the development of an associated theory for the equilibrium of spatial lattices and the derivation and analysis of certain necessary conditions for stable configurations in the presence of configuration-independent conservative loads. In particular, we obtain the second variation of the energy for equilibrium states and use it to generate pointwise necessary conditions for stable equilibria. These are the counterparts of the well known Legendre inequalities associated with one-dimensional variational problems [2].

Section 2 is devoted to a brief discussion of the kinematical and constitutive foundations of the rod theory under consideration. The framework used here generalizes the viewpoint adopted in [3] for inextensible rods. In Section 3 we obtain expressions for the first and second variations of the strain energy based on a local characterization of kinematically admissible configurations. The attendant analysis is complicated by the stringent kinematical restrictions embodied in the theory.

The results are used in Section 4 to obtain the Euler equations of equilibrium. These are identical in form to the well known statical equations of rod theory. This correspondence in turn lends support to the various hypotheses upon which the theory is based. We also derive 
the equilibrium conditions at the nodes of the lattice associated with various types of nodal constraint. These, too, correspond to the conditions that would be obtained from elementary considerations. Finally, in Section 5 we present a detailed derivation of the Legendre necessary condition associated with locally stable configurations. We demonstrate that, contrary to expectation, this condition does not encompass the Legendre condition for elastic cables in the limit of perfect flexibility.

\section{Kinematics and the Basic Constitutive Hypothesis}

Configurations of spatial rods are defined by mappings of an arclength parameter $s \in[0, L]$ onto $\left\{\mathbf{r}(s) \mathbf{e}_{i}(s)\right\} ; i=1,2,3$, where $L$ is the total arclength in a reference placement, $\mathbf{r}(\cdot)$ is the position vector of points on the rod relative to a fixed origin, and the $\mathbf{e}_{i}(\cdot)$ are vectorvalued functions that specify the orientations of cross-sections $s=$ const. The material rod is identified with the reference configuration in which the functions $\mathbf{r}(\cdot)$ and $\mathbf{e}_{i}(\cdot)$ take the values $\mathbf{x}(\cdot)$ and $\mathbf{E}_{i}(\cdot)$, respectively. We take $\left\{\mathbf{E}_{i}\right\}$ to be an orthonormal set for every $s \in[0, L\}$, and identify $\mathbf{E}_{1}(\cdot)$ with the unit tangent to the space curve defined by $\mathbf{x}(\cdot): \mathbf{E}_{1}(\cdot)=\mathbf{x}^{\prime}(\cdot)$. Here and elsewhere, primes are used to denote derivatives with respect to $s$. The vectors $\mathbf{E}_{2}(s)$ and $\mathbf{E}_{3}(s)$ span the plane normal to the curve at arclength station $s$. In principle, there is no further restriction on the specification of these vectors, but it is frequently advantageous to define them so that $\mathbf{E}_{1} \cdot \mathbf{E}_{2} \times \mathbf{E}_{3}=1$, with $\mathbf{E}_{2}$ and $\mathbf{E}_{3}$ along the geometric principal axes of the cross-section.

We consider a constrained theory in which the set $\left\{\mathbf{e}_{i}\right\}$ is orthonormal at every crosssection, with $\mathbf{e}_{1} \cdot \mathbf{e}_{2} \times \mathbf{e}_{3}=1$, and further require that $\mathbf{e}_{1}$ coincide with the unit tangent, $\mathbf{t}$, to the space curve defined by $\mathbf{r}(\cdot)$. Thus

$$
\mathbf{r}^{\prime}(s)=\lambda \mathbf{t}
$$

where

$$
\lambda=\left|\mathbf{r}^{\prime}\right|
$$

is the local stretch of the rod. Moreover, the constraints on $\left\{\mathbf{E}_{i}\right\}$ and $\left\{\mathbf{e}_{i}\right\}$ imply the existence of a rotation $\mathbf{R}(s)$ such that

$$
\mathbf{e}_{i}(s)=\mathbf{R}(s) \mathbf{E}_{i}(s)
$$

In particular

$$
\mathbf{R}=\mathbf{R 1}=\mathbf{R}\left(\mathbf{E}_{i} \otimes \mathbf{E}_{i}\right)=\left(\mathbf{R E}_{i}\right) \otimes \mathbf{E}_{i}=\mathbf{e}_{i} \otimes \mathbf{E}_{i}
$$

where $\mathbf{1}$ is the unit tensor for 3-space.

A kinematical framework of this type is appropriate when modelling the response of thin extensible rods in which shear of the cross-section, relative to the rod axis, is suppressed. It is equivalent to a certain special version of the director-theory of rods, about which much has becn written. We refer to Antman [1] for comprehensive discussion and bibliography.

The kinematical description is completed by introducing a tensor $\mathbf{W}$ defined by:

$$
\mathbf{W}=\mathbf{e}_{i}^{\prime} \otimes \mathbf{e}_{i}=W_{i j} \mathbf{e}_{i} \otimes \mathbf{e}_{j} ; \quad W_{i j}=\mathbf{e}_{i} \cdot \mathbf{e}_{j}^{\prime} .
$$


This furnishes the rate of change of $\left\{\mathbf{e}_{i}\right\}$ with respect to $s$ :

$$
\mathbf{c}_{\imath}^{\prime}=\mathbf{W} \mathbf{c}_{i}
$$

The orthonormality of $\left\{\mathbf{e}_{i}\right\}$ implies that $\mathbf{W}$ is skew, i.e. $\mathbf{W}^{T}=-\mathbf{W}$. Thus $\mathbf{W}(s)$ is equivalent to a vector-valued function $\mathbf{w}(s)$, in the sense that $\mathbf{W} \mathbf{u}=\mathbf{w} \times \mathbf{u}$ for any vector $\mathbf{u}$. The use of w allows (2.6) to be written in the form

$$
\mathbf{e}_{i}^{\prime}=\mathbf{w} \times \mathbf{e}_{i}
$$

The relation between the components $w_{i}\left(=\mathbf{w} \cdot \mathbf{e}_{i}\right)$ and $W_{i j}$ is well known:

$$
w_{\imath}=\frac{1}{2} e_{i j k} W_{k j}, \quad W_{k j}=w_{i} e_{i j k},
$$

where $e_{i j k}$ is the usual permutation symbol $\left(e_{123}=+1\right)$.

It follows from (2.7) that the components $w_{\alpha}(\alpha \in\{2,3\})$ account for the rate of change of the unit tangent $\mathbf{t}\left(=\mathbf{e}_{1}\right)$ with respect to $s$, while $w_{1}$ measures the projection onto the cross-section of the rate of change of the cross-sectional orientation.

Let $\Omega$ be the skew tensor defined by

$$
\boldsymbol{\Omega}=\mathbf{R}^{T} \mathbf{W} \mathbf{R}=W_{i \jmath} \mathbf{E}_{\imath} \otimes \mathbf{E}_{\jmath}
$$

and let

$$
\kappa=\kappa_{i} \mathbf{E}_{i}
$$

be its vector-equivalent:

$$
\kappa_{i}=\frac{1}{2} e_{i j k} W_{k j}
$$

Evidently $\kappa_{i}=w_{i}$ and $\mathbf{w}=\mathbf{R} \boldsymbol{\kappa}$.

It is straightforward to show that $\lambda$ and $\kappa$ are invariant under superimposed rigid deformations

$$
\mathbf{r}(s) \rightarrow \mathbf{Q} \mathbf{r}(s)+\mathbf{c}, \quad \mathbf{e}_{i}(s) \rightarrow \mathbf{Q} \mathbf{e}_{i}(s), \quad \mathbf{e}_{i}^{\prime}(s) \rightarrow \mathbf{Q} \mathbf{e}_{i}^{\prime}(s),
$$

where $\mathbf{Q}$ is an arbitrary fixed rotation and $\mathbf{c}$ is an arbitrary fixed vector. Because of this invariance property, it is natural to formulate a theory for elastic rods by introducing a strain energy, $w$, per unit length of the reference placement, that depends on $\lambda$ and $\kappa$ :

$$
w=w(\lambda, \kappa)
$$

Here we suppress reference to possible dependence on arclength $s$ that may arise due to nonuniformity of the material properties, or to the presence of non-zero values of the functions

$$
\kappa_{i}^{0}(s)=\frac{1}{2} e_{i j k} \mathbf{E}_{k} \cdot \mathbf{E}_{j}^{\prime} .
$$

These are the values of $\kappa_{i}(s)$ in the configuration $\left\{\mathbf{x}, \mathbf{E}_{i}\right\}$. It is often desirable to include them explicitly as parameters in the strain energy function $[3,4]$. We leave any such dependence tacit, as it does not affect the considerations of this work. 
We remark that the form (2.13) may be deduced from the fundamental postulate that the strain energy depends on the variables $\mathbf{r}^{\prime}, \mathbf{e}_{i}$, and $\mathbf{e}_{i}^{\prime}$, together with (2.1) and the assumption of invariance of the energy under superimposed rigid motions [1]. A thorough discussion of this point may be found in [1], which includes developments of theories that are more general than the one considered here. References $[1,3]$ also contain descriptions of the notion of material symmetry in this context.

$\Lambda$ model of elastic cables may be obtained by eliminating $\kappa_{i}$ (and $\kappa_{i}^{0}$ ) from the list of arguments of the strain energy function. Alternatively, if dependence on $\lambda$ is eliminated (i.e. by setting $\lambda \equiv 1$ ), we recover the theory of inextensible rods considered in [3]. Retention of the full list enables us to consider a wide range of problems using a single theory.

Finally, we note that Dill [5] has recently presented an in-depth analysis of the relationship between the present theory and the three-dimensional theory of elasticity for isotropic solids.

\section{The Total Strain Energy and its First and Second Variations}

The strain energy of the rod is the functional of the configuration $\left\{\mathbf{r}, \mathbf{e}_{i}\right\}$ defined by

$$
S=\int_{0}^{L} w(\lambda, \kappa) \mathrm{d} s
$$

Let $\varepsilon \in\left(-\varepsilon_{o}, \varepsilon_{o}\right)$ for some positive number $\varepsilon_{o}$, and consider a smooth one-parameter family of kinematically admissible configurations $\left\{\mathbf{r}^{*}(s ; \varepsilon), \mathbf{e}_{i}^{*}(s ; \varepsilon)\right\}$, with $\left\{\mathbf{r}^{*}(s ; 0), \mathbf{e}_{i}^{*}(s ; 0)\right\}=$ $\left\{\mathbf{r}(s), \mathbf{e}_{i}(s)\right\}$. Here kinematic admissibility means that, for each fixed $\varepsilon, \mathbf{r}^{*}(\cdot ; \varepsilon)$ and $\mathbf{e}_{i}^{*}(\cdot ; \varepsilon)$ are at least piecewise $C^{2}$ on $[0, L]$ and satisfy (2.1) and (2.2):

$$
\mathbf{r}^{*^{\prime}}=\lambda^{*} \mathbf{t}^{*} ; \quad \lambda^{*}=\left|\mathbf{r}^{*^{\prime}}\right|, \quad \mathbf{t}^{*}=\mathbf{e}_{1}^{*} .
$$

It is possible to relax the continuity hypothesis if equilibria with discontinuities in $\lambda$ or $\kappa$ are of interest [3], but we do not consider such examples here. Further conditions regarding kinematic admissibility are introduced in Section 4 as required.

Let superimposed dots denote derivatives of functions with respect to $\varepsilon$, evaluated at $\varepsilon=0$. Then the first and second variations of $S$ at the configuration $\left\{\mathbf{r}, \mathbf{e}_{i}\right\}$ are

$$
\dot{S}=\int_{0}^{L}\left[(\partial w / \partial \lambda) \dot{\lambda}+\left(\partial w / \partial \kappa_{i}\right) \dot{\kappa}_{i}\right] \mathrm{d} s
$$

and

$$
\ddot{S}=\int_{0}^{L}\left[\left(\partial^{2} w / \partial \lambda^{2}\right)(\dot{\lambda})^{2}+(\partial w / \partial \lambda) \ddot{\lambda}+2 D_{\imath} \dot{\kappa}_{i} \dot{\lambda}+C_{i j} \dot{\kappa}_{i} \dot{\kappa}_{j}+M_{i} \ddot{\kappa}_{i}\right] \mathrm{d} s
$$

respectively, where

$$
M_{i}=\partial w / \partial \kappa_{i}, \quad D_{i}=\partial^{2} w / \partial \lambda \partial \kappa_{i}, \quad C_{i j}=\partial^{2} w / \partial \kappa_{i} \partial \kappa_{j}
$$

and all the derivatives of $w(\cdot, \cdot)$ are evaluated at $\varepsilon=0$. Here $\left\{\dot{\lambda}, \dot{\kappa}_{i}\right\}$ and $\left\{\ddot{\lambda}, \ddot{\kappa}_{i}\right\}$ are the first and second variations of $\left\{\lambda, \kappa_{i}\right\}$ induced by the variations of $\left\{\mathbf{r}^{*}, \mathbf{e}_{i}^{*}\right\}$.

To analyze the structure of these variations near $\varepsilon=0$, we write

$$
\mathbf{e}_{i}^{*}(s ; \varepsilon)=\mathbf{e}_{i}(s)+\varepsilon \dot{\mathbf{e}}_{i}(s)+\frac{1}{2} \varepsilon^{2} \ddot{\mathbf{e}}_{i}(s)+o\left(\varepsilon^{2}\right),
$$


and

$$
\mathbf{r}^{*}(s ; \varepsilon)=\mathbf{r}(s)+\varepsilon \mathbf{u}(s)+\frac{1}{2} \varepsilon^{2} \mathbf{v}(s)+o\left(\varepsilon^{2}\right)
$$

where

$$
\mathbf{u}=\dot{\mathbf{r}} \quad \text { and } \quad \mathbf{v}=\ddot{\mathbf{r}}
$$

Let $\mathbf{R}^{*}(s ; \varepsilon)$ be the rotation that maps $\left\{\mathbf{E}_{i}\right\}$ onto $\left\{\mathbf{e}_{i}^{*}\right\}: \mathbf{R}^{*}=\mathbf{e}_{i}^{*} \otimes \mathbf{E}_{i}$. Then

$$
\frac{\mathrm{d}}{\mathrm{d} \varepsilon} \mathbf{e}_{i}^{*}=\boldsymbol{\alpha}^{*} \mathbf{e}_{i}^{*}=\mathbf{a}^{*} \times \mathbf{e}_{i}^{*}
$$

where

$$
\boldsymbol{\alpha}^{*}(s ; \varepsilon)=\left(\frac{\mathrm{d}}{\mathrm{d} \varepsilon} \mathbf{R}^{*}\right)\left(\mathbf{R}^{*}\right)^{T}
$$

is a skew tensor and $\mathbf{a}^{*}(s ; \varepsilon)$ is its vector-equivalent. Consequently,

$$
\dot{\mathbf{e}}_{i}=\mathbf{a} \times \mathbf{e}_{i}
$$

where $\mathbf{a}(s)=\mathbf{a}^{*}(s ; 0)$. Further differentiation of (3.9) yields

$$
\ddot{\mathbf{e}}_{i}=\mathbf{a} \times\left(\mathbf{a} \times \mathbf{e}_{i}\right)+\mathbf{b} \times \mathbf{e}_{i}
$$

where $\mathbf{b}(s)=\left.(\mathbf{d} / \mathrm{d} \varepsilon) \mathbf{a}^{*}\right|_{\varepsilon=0}$.

Next, we define the scalar functions

$$
a(s)=\dot{\lambda}, \quad b(s)=\ddot{\lambda}
$$

so that

$$
\lambda^{*}(s ; \varepsilon)=\lambda(s)+\varepsilon a(s)+\frac{1}{2} \varepsilon^{2} b(s)+o\left(\varepsilon^{2}\right) .
$$

On combining this with (3.6), (3.7) and the constraint (3.2), we obtain the compatibility conditions

$$
\mathbf{u}^{\prime}(s)=a \mathbf{t}+\lambda \mathbf{a} \times \mathbf{t}=a \mathbf{t}+\mathbf{a} \times \mathbf{r}^{\prime}
$$

and

$$
\mathbf{v}^{\prime}(s)=2 a(\mathbf{a} \times \mathbf{t})+\mathbf{a} \times\left(\mathbf{a} \times \mathbf{r}^{\prime}\right)+b \mathbf{t}+\mathbf{b} \times \mathbf{r}^{\prime}
$$

Formulae for the first and second variations of $\kappa_{i}^{*}(s ; \varepsilon)$ may be derived from (2.5), (2.11) and (3.11):

$$
\begin{aligned}
\dot{\kappa}_{i} & =\frac{1}{2} e_{i j k}\left(\dot{\mathbf{e}}_{k} \cdot \mathbf{e}_{j}^{\prime}+\mathbf{e}_{k} \cdot \dot{\mathbf{e}}_{j}^{\prime}\right) \\
& =\frac{1}{2} e_{2 j k}\left[\mathbf{a} \times \mathbf{e}_{k} \cdot \mathbf{e}_{j}^{\prime}+\mathbf{e}_{k} \cdot\left(\mathbf{a}^{\prime} \times \mathbf{e}_{j}+\mathbf{a} \times \mathbf{e}_{j}^{\prime}\right)\right]
\end{aligned}
$$


The terms involving a cancel, and we obtain

$$
\dot{\kappa}_{i}=\frac{1}{2} e_{i j k} \mathbf{a}^{\prime} \cdot \mathbf{e}_{j} \times \mathbf{e}_{k}=\mathbf{a}^{\prime} \cdot\left(\frac{1}{2} e_{i j k} e_{j k m} \mathbf{e}_{m}\right) .
$$

One of the $e-\delta$ identities then furnishes the result

$$
\dot{\kappa}_{i}=\mathbf{e}_{i} \cdot \mathbf{a}^{\prime}
$$

With the use of (3.12), we may also show that

$$
\ddot{\kappa}_{i}=\mathbf{e}_{i} \cdot \mathbf{a}^{\prime} \times \mathbf{a}+\mathbf{e}_{i} \cdot \mathbf{b}^{\prime}
$$

Let $x(s)$ and $\mathbf{y}(s)$ be piecewise $C^{2}$ scalar-valued and vector-valued functions, respectively. We define

$$
I\left[x, \mathbf{y}^{\prime}\right]=\int_{0}^{L}\left[(\partial w / \partial \lambda) x+\mathbf{M} \cdot \mathbf{y}^{\prime}\right] \mathrm{d} s
$$

and

$$
J\left[x, \mathbf{y}, \mathbf{y}^{\prime}\right]=\int_{0}^{L}\left[\left(\partial^{2} w / \partial \lambda^{2}\right) x^{2}+\mathbf{y}^{\prime} \cdot \mathbf{C} \mathbf{y}^{\prime}+\mathbf{M} \cdot \mathbf{y}^{\prime} \times \mathbf{y}+2 x \mathbf{D} \cdot \mathbf{y}^{\prime}\right] \mathrm{d} s
$$

where

$$
\mathbf{M}=M_{i} \mathbf{e}_{i}, \quad \mathbf{D}=D_{i} \mathbf{e}_{i}, \quad \mathbf{C}=C_{i j} \mathbf{e}_{i} \otimes \mathbf{e}_{j}
$$

and $M_{i}, D_{i}, C_{i j}$ are defined in (3.5). In the next section we interpret $\mathbf{M}(s)$ as the moment exerted on the part $[0, s]$ of the rod by the remainder $(s, L]$.

Substitution of (3.13) and (3.19-23) into (3.3) and (3.4) yields the first and second variations of the strain energy in the forms

$$
\dot{S}=I\left[a, \mathbf{a}^{\prime}\right] \quad \text { and } \quad \ddot{S}=I\left[b, \mathbf{b}^{\prime}\right]+J\left[a, \mathbf{a}, \mathbf{a}^{\prime}\right]
$$

\section{Lattices}

Consider a collection of $n$ rods. Let the $j$ th rod have arclength $L_{j}$ in its reference configuration. Suppose these are joined together at $l$ nodes located at the positions $\mathbf{x}_{k} ; k=1, \ldots, l$. After deformation, the nodes displace to the (unknown) positions $\mathbf{y}_{k}$. Let the collection of index labels of these nodes be the set $K$. At each node $k \in K$, a dead load $\mathbf{q}_{k}$ is prescribed. In addition, we suppose that $m$ nodes are fixed at the locations $\mathbf{z}_{h} ; h=1, \ldots, m$. Their labels belong to the set $H$. The collection of all node labels is $K \cup H$.

Let $s_{j} \in\left[0, L_{j}\right]$ measure reference arclength along the $j$ th rod. The position function in a typical configuration is $\mathbf{r}_{j}\left(s_{j}\right)$ and the orientation triad is $\left\{\mathbf{e}_{\imath}\left(s_{j}\right)\right\}_{j}$. Following Cannarozzi [6], we introduce the sets

$$
\begin{aligned}
& I_{k}=\left\{j: s_{j}=0 \quad \text { at node } k \in K\right\}, \\
& E_{k}=\left\{j: s_{j}=L_{j} \text { at node } k \in K\right\}
\end{aligned}
$$


and

$$
\begin{aligned}
& I_{h}=\left\{j: s_{j}=0 \quad \text { at node } h \in H\right\}, \\
& E_{h}=\left\{j: s_{j}=L_{j} \text { at node } h \in H\right\} .
\end{aligned}
$$

Henceforth we use superscripts 0 and $L$ to denote the values of functions at $s_{j}=0$ and $s_{\jmath}=L_{j}$, respectively. Thus

$$
\mathbf{r}_{j}^{0}=\mathbf{r}_{j}(0) \quad \text { and } \quad \mathbf{r}_{j}^{L}=\mathbf{r}_{j}\left(L_{j}\right)
$$

These are subject to the conditions

$$
\mathbf{r}_{j}^{0}=\mathbf{y}_{k}, \quad j \in I_{k} ; \quad \mathbf{r}_{j}^{L}=\mathbf{y}_{k}, \quad j \in E_{k}
$$

and

$$
\mathbf{r}_{j}^{0}=\mathbf{z}_{h}, \quad j \in I_{h} ; \quad \mathbf{r}_{j}^{L}=\mathbf{z}_{h}, \quad j \in E_{h},
$$

which ensure the continuity of the lattice at the nodes.

The potential energy, $E$, of a configuration of the entire lattice is

$$
E=\sum_{j=1}^{n} S_{j}-\sum_{k=1}^{l} \mathbf{q}_{k} \cdot \mathbf{y}_{k}
$$

where $S_{\jmath}$ is the total strain energy of the $j$ th rod. A configuration of the lattice is equilibrated if and only if the associated first variation of the energy vanishes for all admissible variations of the kinematical variables:

$$
0=\sum_{j=1}^{n} \dot{S}_{j}-\sum_{k=1}^{l} \mathbf{q}_{k} \cdot \mathbf{u}_{k} ; \quad \mathbf{u}_{k}=\dot{\mathbf{y}}_{k},
$$

where

$$
\dot{S}_{j}=\int_{0}^{L_{J}}\left[(\partial w / \partial \lambda) \mathbf{t} \cdot \mathbf{u}^{\prime}+\mathbf{M} \cdot \mathbf{a}^{\prime}\right] \mathrm{d} s
$$

Here (3.13) 1 and (3.15) have been invoked and the index $j$ has been suppressed in the integrand for the sake of clarity.

To proceed it is necessary to account explicitly for the fact that the variations $\mathbf{u}(s)$ and $\mathbf{a}(s)$ are not independent. In particular, scalar multiplication of (3.15) with $\mathbf{e}_{\alpha}(\alpha=2,3)$ furnishes the constraints:

$$
\mathbf{u}^{\prime} \cdot \mathbf{e}_{\alpha}+\mathbf{r}^{\prime} \cdot \mathbf{a} \times \mathbf{e}_{\alpha}=0 ; \quad \alpha=2,3 .
$$

When these are satisfied, $\dot{S}_{j}$ coincides with the augmented functional

$$
\begin{aligned}
T_{j} & =\int_{0}^{L_{3}}\left[(\partial w / \partial \lambda) \mathbf{t} \cdot \mathbf{u}^{\prime}+\mathbf{M} \cdot \mathbf{a}^{\prime}+F_{\alpha}\left(\mathbf{u}^{\prime} \cdot \mathbf{e}_{\alpha}+\mathbf{r}^{\prime} \cdot \mathbf{a} \times \mathbf{e}_{\alpha}\right)\right] \mathrm{d} s \\
& =\int_{0}^{L_{3}}\left[\mathbf{F} \cdot \mathbf{u}^{\prime}+\mathbf{M} \cdot \mathbf{a}^{\prime}+\mathbf{r}^{\prime} \cdot \mathbf{a} \times\left(F_{\alpha} \mathbf{e}_{\alpha}\right)\right] \mathrm{d} s,
\end{aligned}
$$


where $F_{\alpha}(s), \alpha \in\{2,3\}$, are Lagrange multipliers, and

$$
\mathbf{F}=(\partial w / \partial \lambda) \mathbf{t}+F_{\alpha} \mathbf{e}_{\alpha} .
$$

Since $\mathbf{r}^{\prime}$ is parallel to $\mathbf{t}$, the last term in (4.1) may be replaced with $\mathbf{a} \cdot \mathbf{F} \times \mathbf{r}^{\prime}$, and integration by parts yields

$$
T_{j}=U_{j}+\mathbf{F}_{j}^{L} \cdot \mathbf{u}_{j}^{L}+\mathbf{M}_{j}^{L} \cdot \mathbf{a}_{j}^{L}-\mathbf{F}_{j}^{0} \cdot \mathbf{u}_{j}^{0}-\mathbf{M}_{j}^{0} \cdot \mathbf{a}_{j}^{0},
$$

with

$$
U_{j}=-\int_{0}^{L_{3}}\left[\mathbf{u} \cdot \mathbf{F}^{\prime}+\mathbf{a} \cdot\left(\mathbf{M}^{\prime}-\mathbf{F} \times \mathbf{r}^{\prime}\right)\right] \mathrm{d} s .
$$

These expressions may be used to reduce the stationary-energy condition, eq. (4.7), to

$$
\begin{aligned}
0= & \sum_{j=1}^{n} U_{j}-\sum_{k=1}^{l} \mathbf{u}_{k} \cdot\left[\mathbf{q}_{k}-\left(\sum_{j \in E_{k}} \mathbf{F}_{j}^{L}-\sum_{j \in I_{k}} \mathbf{F}_{j}^{0}\right)\right] \\
& +\sum_{k=1}^{l}\left(\sum_{j \in E_{k}} \mathbf{M}_{j}^{L} \cdot \mathbf{a}_{j}^{L}-\sum_{j \in I_{k}} \mathbf{M}_{j}^{0} \cdot \mathbf{a}_{j}^{0}\right) \\
& +\sum_{h=1}^{m}\left(\sum_{j \in E_{h}} \mathbf{M}_{j}^{L} \cdot \mathbf{a}_{\jmath}^{L}-\sum_{\jmath \in I_{h}} \mathbf{M}_{j}^{0} \cdot \mathbf{a}_{j}^{0}\right),
\end{aligned}
$$

wherein we have imposed the constraints

$$
\begin{aligned}
& \mathbf{u}_{j}^{0}=\mathbf{u}_{k}, \quad j \in I_{k} ; \quad \mathbf{u}_{j}^{L}=\mathbf{u}_{k}, \quad j \in E_{k} ; \\
& \mathbf{u}_{j}^{0}=\mathbf{0}, \quad j \in I_{h} ; \quad \mathbf{u}_{j}^{L}=\mathbf{0}, \quad j \in E_{h} .
\end{aligned}
$$

Restrictions on the virtual rotations $\mathbf{a}_{j}^{0}$ and $\mathbf{a}_{j}^{L}$ must also be imposed in accordance with the particular type of nodal connection under consideration.

Now (4.14) must be satisfied for all admissible $\mathbf{u}_{k}, \mathbf{a}_{j}^{0}$ and $\mathbf{a}_{j}^{L}$. Null values of these variations are admissible in all lattice types, and for this choice (4.14) requires that the sum $\sum U_{j}$ vanish. By choosing $\mathbf{u}(s)$ and $\mathbf{a}(s)$ to be non-zero in each of the $n$ rods in succession, we conclude that $U_{j}=0 ; j=1, \cdots, n$, where $U_{j}$ is given by (4.13). The multiplier rule [2] of the calculus of variations then yields the equilibrium equations in each rod:

$$
\mathbf{F}^{\prime}=\mathbf{0}, \quad \mathbf{M}^{\prime}=\mathbf{F} \times \mathbf{r}^{\prime} .
$$

These are identical to the classical equations of rod theory [5], in the absence of distributed load, provided that $\mathbf{F}(s)$ and $\mathbf{M}(s)$ are identified with the force and moment, respectively, exerted by the part $[s, L]$ on the remainder $[0, s)$. (Conversely, $-\mathbf{F}(s)$ and $-\mathbf{M}(s)$ are the force and moment exerted by $[0, s]$ on $(s, L])$. The moment is given by the constitutive equation (3.5) (with (3.23)). In view of (4.11), it is only the tangential component of the force that is determined by a constitutive equation. The transverse components $F_{\alpha}$ are shear reactions that 
are workless in any variation of the configuration compatible with (4.9). They are determined by equilibrium considerations alone.

We remark here that Tadjbakhsh and Lagoudas [7] recently presented an alternative variational treatment of a dynamical theory for rods of the kind considered here. In their work, the counterpart of eq. (2.1) (or (3.15)) is treated a constraint in its entirety, and all three components of the force $\mathbf{F}$ are regarded as Lagrange multipliers. The tangential component is then given by a constitutive equation a posteriori. However, this is unnecessary because the variation $\dot{\lambda}(=a)$ of the local stretch is not subject to any restriction. Thus, according to (3.15), the tangential part of $\mathbf{u}^{\prime}$ is arbitrary. It is only the transverse components that are restricted, in accordance with (4.9). Equation (2.1) may be interpreted as a constraint if the rod is inextensible $(\lambda \equiv 1)$. In this case all components of the force are constitutively-indeterminate (e.g. [3]).

With (4.16) satisfied in each rod, all of the $U_{j}$ in (4.14) vanish, and the remaining expression must be satisfied for all $\mathbf{u}_{k}$ and for all admissible $\mathbf{a}_{j}^{0}$ and $\mathbf{a}_{j}^{L}$. On setting $\mathbf{a}_{j}^{0}=\mathbf{0}, \mathbf{a}_{j}^{L}=\mathbf{0}$ and taking all but one of the $\mathbf{u}_{k}$ to be zero in succession, we obtain the nodal force balance equations

$$
\sum_{\jmath \in E_{k}} \mathbf{F}_{\jmath}^{L}-\sum_{j \in I_{k}} \mathbf{F}_{j}^{0}=\mathbf{q}_{k}, \quad k \in K .
$$

These require that the net effect of the nodal reactions (the forces exerted by the nodes on the rods) be such as to balance the applied forces.

Additional equilibrium conditions may be derived, but these vary depending on the class of nodal constraint. We present discussions of three of these classes.

\section{(a) Unrestricted rotations}

If the rod rotations are unrestricted at the nodes, then there are no kinematical constraints on virtual displacements or rotations beyond those that have already been imposed. Nodal connections of this type are appropriate in a theory of cable networks, or in models of ball and socket joints in structural lattices.

For illustrative purposes, suppose that a particular node $k^{\prime} \in K$ is of this type. Then a necessary condition for $(4.14)$ is

$$
\sum_{j \in E_{k^{\prime}}} \mathbf{M}_{j}^{L} \cdot \mathbf{a}_{j}^{L}-\sum_{j \in I_{k^{\prime}}} ; \mathbf{M}_{j}^{0} \cdot \mathbf{a}_{j}^{0}=\mathbf{0} .
$$

For each $j \in E_{k^{\prime}} \cup I_{k^{\prime}}$, the $\mathbf{a}_{j}^{0}$ and $\mathbf{a}_{j}^{L}$ may be specified independently, so the node is equilibrated only if it transmits no moment to any of the attached rods:

$$
\mathbf{M}_{j}^{0}=\mathbf{0}, \quad j \in I_{k^{\prime}} ; \quad \mathbf{M}_{j}^{L}=\mathbf{0}, \quad j \in E_{k^{\prime}} .
$$

\section{(b) Rigidly constrained rotations}

Once again we focus attention on a particular node $k^{\prime} \in K$ for the sake of illustration. Suppose the nodal connection is rigid in the sense that, for any two values of $j$ in the set $I_{k^{\prime}} \cup E_{k^{\prime}}$, the angles formed by the triads $\left\{\mathbf{E}_{i}\right\}_{j}^{0}$ and $\left\{\mathbf{E}_{i}\right\}_{j}^{L}$ are preserved under deformation. This is equivalent to requiring that

$$
\mathbf{R}_{j}^{0}=\mathbf{R}_{k^{\prime}}, \quad j \in I_{k^{\prime}} ; \quad \mathbf{R}_{\jmath}^{L}=\mathbf{R}_{k^{\prime}}, \quad j \in E_{k^{\prime}}
$$


for some rotation $\mathbf{R}_{k^{\prime}}$, where $\mathbf{R}^{\circ}$ and $\mathbf{R}^{L}$ are the endpoint values of the rod rotation $\mathbf{R}$ defined in (2.4). If such conditions are imposed in all configurations, then (3.10) may be used to derive an associated set of restrictions on the virtual endpoint rotations:

$$
\mathbf{a}_{j}^{0}=\mathbf{a}_{k^{\prime}}, \quad j \in I_{k^{\prime}} ; \quad \mathbf{a}_{j}^{L}=\mathbf{a}_{k^{\prime}}, \quad j \in E_{k^{\prime}},
$$

for some arbitrary vector $\mathbf{a}_{k^{\prime}}$.

In the present case (4.18) remains valid, and (4.21) yields

$$
\mathbf{a}_{k^{\prime}} \cdot \boldsymbol{\mu}_{k^{\prime}}=0 \quad \text { for all } \mathbf{a}_{k^{\prime}},
$$

where

$$
\boldsymbol{\mu}_{k^{\prime}}=\sum_{j \in E_{k^{\prime}}} \mathbf{M}_{\jmath}^{L}-\sum_{j \in I_{k^{\prime}}} \mathbf{M}_{j}^{0} .
$$

Thus the net moment at the node vanishes: $\boldsymbol{\mu}_{k^{\prime}}=\mathbf{0}$.

(c) Concurrent axes of rotation

As a final example, let the rods joined at node $k^{\prime} \in K$ be constrained in such a way as to pivot freely about a common axis with orientation $\mathbf{N}$ in the reference configuration of the lattice. Then the endpoint rotations $\mathbf{R}_{j}^{0}$ and $\mathbf{R}_{j}^{L}$ are such that

$$
\mathbf{R}_{j}^{0} \mathbf{N}=\mathbf{n}, \quad j \in I_{k^{\prime}} ; \quad \mathbf{R}_{j}^{L} \mathbf{N}=\mathbf{n}, \quad j \in E_{k^{\prime}},
$$

where $\mathbf{n}$ is the pivotal axis in the deformed lattice. We take $\mathbf{N}$ and $\mathbf{n}$ to be unit vectors without loss of generality.

The variational versions of (4.24) are

$$
\mathbf{a}_{\jmath}^{0} \times \mathbf{n}=\boldsymbol{\beta}, \quad j \in I_{k^{\prime}} ; \quad \mathbf{a}_{j}^{L} \times \mathbf{n}=\boldsymbol{\beta}, \quad j \in E_{k^{\prime}},
$$

where

$$
\boldsymbol{\beta}=\dot{\mathbf{n}}
$$

is the variation of $\mathbf{n}$. We note that $\boldsymbol{\beta} \cdot \mathbf{n}=0$ because $\mathbf{n}$ is a unit vector. Now any vector a may be represented in the form

$$
\mathbf{a}=(\mathbf{n} \cdot \mathbf{a}) \mathbf{n}+\mathbf{n} \times(\mathbf{a} \times \mathbf{n}),
$$

so that

$$
\mathbf{a}_{j}^{0}=\alpha_{j}^{0} \mathbf{n}+\mathbf{n} \times \boldsymbol{\beta} \quad \text { and } \quad \mathbf{a}_{j}^{L}=\alpha_{j}^{L} \mathbf{n}+\mathbf{n} \times \boldsymbol{\beta}
$$

for some scalars $\alpha_{j}^{0}, \alpha_{j}^{L}$.

Substitution of (4.28) into (4.18) yields

$$
0=\sum_{\jmath \in E_{k^{\prime}}} \alpha_{j}^{L} \mathbf{n} \cdot \mathbf{M}_{j}^{L}-\sum_{j \in I_{k^{\prime}}} \alpha_{j}^{0} \mathbf{n} \cdot \mathbf{M}_{\jmath}^{0}+\mathbf{n} \times \boldsymbol{\beta} \cdot \boldsymbol{\mu}_{k^{\prime}},
$$


where $\boldsymbol{\mu}_{k^{\prime}}$ is given by (4.23). This must hold for arbitrary $\alpha_{j}^{0}, \alpha_{j}^{L}$, and for any $\beta$ perpendicular to $\mathbf{n}$. By setting $\beta=\mathbf{0}$ and all but one of the $\alpha$ 's equal to zero in succession, we derive the necessary conditions

$$
\mathbf{n} \cdot \mathbf{M}_{j}^{0}=0, \quad j \in I_{k^{\prime}} ; \quad \mathbf{n} \cdot \mathbf{M}_{j}^{L}=0, \quad j \in E_{k^{\prime}} .
$$

Thus the node transmits no axial moment to any of the attached rods. Finally, the last term in (4.29) requires that $\boldsymbol{\mu}_{k^{\prime}}$ be orthogonal to any vector in the plane perpendicular to $\mathbf{n}$, so that $\boldsymbol{\mu}_{k^{\prime}}=\left(\mathbf{n} \cdot \boldsymbol{\mu}_{k^{\prime}}\right) \mathbf{n}$. But

$$
\mathbf{n} \cdot \boldsymbol{\mu}_{k^{\prime}}=\sum_{j \in E_{k^{\prime}}} \mathbf{n} \cdot \mathbf{M}_{j}^{L}-\sum_{j \in I_{k^{\prime}}} \mathbf{n} \cdot \mathbf{M}_{\jmath}^{0},
$$

and this vanishes by (4.30). Thus there is no net moment at the node:

$$
\mu_{k^{\prime}}=\mathbf{0}
$$

\section{Further Necessary Conditions for a Minimum of the Energy}

(a) The second variation of the energy at equilibrium

For an equilibrium configuration to be stable, it is necessary that the second variation of the energy, evaluated at that configuration, be nonnegative. In the present context, this is equivalent to the requirement

$$
\sum_{j=1}^{n} \ddot{S}_{j}-\sum_{k=1}^{l} \mathbf{q}_{k} \cdot \mathbf{v}_{k} \geqslant 0
$$

where

$$
\mathbf{v}_{k}=\ddot{\mathbf{y}}_{k} \quad \text { for } k \in K,
$$

and

$$
\ddot{S}_{\jmath}=I_{j}\left[b, \mathbf{b}^{\prime}\right]+J_{j}\left[a, \mathbf{a}, \mathbf{a}^{\prime}\right]
$$

is the second variation of the strain energy of the $j$ th rod (see (3.24)).

To proceed we use the equilibrium conditions derived in the previous section to obtain restrictions on the equilibrium values of the functionals $I_{j}$. Substituting (4.11) and (4.16) into (3.21), we derive

$$
I_{j}=\int_{0}^{L_{j}}\left(b \mathbf{t} \cdot \mathbf{F}-\mathbf{b} \cdot \mathbf{F} \times \mathbf{r}^{\prime}\right) \mathrm{d} s+\mathbf{M}_{j}^{L} \cdot \mathbf{b}_{j}^{L}-\mathbf{M}_{j}^{0} \cdot \mathbf{b}_{j}^{0} .
$$

Here we use the compatibility condition (3.16) to reduce the first term of the integrand to

$$
b \mathbf{t} \cdot \mathbf{F}=\mathbf{F} \cdot \mathbf{v}^{\prime}+\mathbf{b} \cdot \mathbf{F} \times \mathbf{r}^{\prime}-2 a \mathbf{F} \cdot \mathbf{a} \times \mathbf{t}-\mathbf{F} \cdot \mathbf{a} \times\left(\mathbf{a} \times \mathbf{r}^{\prime}\right) .
$$

From $(4.16)_{1}$, the leading term in the latter expression is equal to $(\mathbf{F} \cdot \mathbf{v})^{\prime}$, and we obtain 


$$
\begin{aligned}
I_{\jmath}= & \mathbf{F}_{j}^{L} \cdot \mathbf{v}_{j}^{L}-\mathbf{F}_{j}^{0} \cdot \mathbf{v}_{j}^{0}+\mathbf{M}_{j}^{L} \cdot \mathbf{b}_{j}^{L}-\mathbf{M}_{j}^{0} \cdot \mathbf{b}_{j}^{0} \\
& -\int_{0}^{L_{\jmath}}\left[2 a \mathbf{F} \cdot \mathbf{a} \times \mathbf{t}+\mathbf{F} \cdot \mathbf{a} \times\left(\mathbf{a} \times \mathbf{r}^{\prime}\right)\right] \mathrm{d} s .
\end{aligned}
$$

For the sake of brevity we restrict our further considerations to nodal constraints of the type treated in Section 4(a). Thus we impose (4.19) together with the kinematical conditions

$$
\mathbf{v}_{j}^{0}=\mathbf{v}_{k}, \quad j \in I_{k} ; \quad \mathbf{v}_{j}^{L}=\mathbf{v}_{k}, \quad j \in E_{k} .
$$

On combining (5.3), (5.6) and (3.22), we find that (5.1) is equivalent to

$$
\sum_{j=1}^{n}\left(F_{j}+G_{j}\right)-\sum_{k=1}^{l} \mathbf{v}_{k} \cdot\left[\mathbf{q}_{k}-\left(\sum_{j \in E_{k}} \mathbf{F}_{j}^{L}-\sum_{j \in I_{k}} \mathbf{F}_{j}^{0}\right)\right] \geqslant 0,
$$

where

$$
F_{j}=\int_{0}^{L_{\jmath}} F^{*}\left(a, \mathbf{a}^{\prime}\right) \mathrm{d} s, \quad G_{j}=\int_{0}^{L_{\jmath}} G^{*}\left(a, \mathbf{a}, \mathbf{a}^{\prime}\right) \mathrm{d} s
$$

and

$$
\left.\begin{array}{l}
F^{*}\left(a, \mathbf{a}^{\prime}\right)=\left(\partial^{2} w / \partial \lambda^{2}\right) a^{2}+\mathbf{a}^{\prime} \cdot \mathbf{C} \mathbf{a}^{\prime}+2 a \mathbf{D} \cdot \mathbf{a}^{\prime}, \\
G^{*}\left(a, \mathbf{a}, \mathbf{a}^{\prime}\right)=\mathbf{M} \cdot \mathbf{a}^{\prime} \times \mathbf{a}-2 a \mathbf{F} \cdot \mathbf{a} \times \mathbf{t}-\mathbf{F} \cdot \mathbf{a} \times\left(\mathbf{a} \times \mathbf{r}^{\prime}\right) .
\end{array}\right\}
$$

In view of (4.17), all of the terms in square brackets in (5.8) vanish separately. Thus

$$
\sum_{j}\left(F_{j}+G_{j}\right) \geqslant 0 .
$$

(b) The Legendre inequality

Following the development of the classical necessary conditions for one-dimensional variational problems [2], we consider local variations $a(s)$ and $\mathbf{a}(s)$ that vanish identically in all but one of the rods. In the remaining rod, we consider variations of the form

$$
\mathbf{a}(s)=\mathbf{e} f(s), \quad a(s)=e f^{\prime}(s),
$$

where $\mathbf{e}$ is a fixed vector, $e$ is a constant, and $f(s)$ is the continuous piecewise $C^{1}$ function defined by

$$
f(s)= \begin{cases}s-s_{1}, & s_{1} \leqslant s \leqslant s_{2} \\ -\frac{\theta}{1-\theta}\left(s-s_{3}\right), & s_{2} \leqslant s \leqslant s_{3} \\ 0, & s \in[0, L] \backslash\left(s_{1}, s_{3}\right) .\end{cases}
$$

Here $s_{1}, s_{2}$ and $s_{3}$ are three arbitrarily chosen points in the interval $[0, L]$, with $s_{1}<s_{2}<s_{3}, L$ is the initial length of the rod, and

$$
\theta=\left(s_{2}-s_{1}\right) /\left(s_{3}-s_{1}\right) \in(0,1) .
$$


The induced variation $\mathbf{u}(s)$ may be constructed by integrating (3.15).

We remark that the variations defined by (5.12) do not possess the degree of continuity required in the development leading up to (5.11). To rectify this, one may use the method of mollifiers [8] to construct sequences of $C^{\infty}$ functions that converge boundedly to $(5.12)_{1,2}$. The use of such sequences leads to precisely the same results as those obtained by using (5.12) directly.

For variations of the typc considered, the single non-vanishing term in the sum (5.11) must be nonnegative:

$$
F+G \geqslant 0,
$$

where the functionals $F$ and $G$ are defined in $(5.9,10)$. Let

$$
\Delta=s_{3}-s_{1}, \quad \Delta_{1}=s_{2}-s_{1}=\theta \Delta, \quad \Delta_{2}=s_{3}-s_{2}=(1-\theta) \Delta .
$$

Then

$$
\begin{aligned}
\Delta^{-1} F & =\Delta^{-1} \int_{s_{1}}^{s_{3}} F^{*}\left(a, \mathbf{a}^{\prime}\right) \mathrm{d} s \\
& =\frac{\theta}{\Delta_{1}} \int_{s_{1}}^{s_{2}} F^{*}(e, \mathbf{e}) \mathrm{d} s+\frac{1-\theta}{\Delta_{2}} \int_{s_{2}}^{s_{3}} F^{*}\left(-\frac{\theta}{1-\theta} e,-\frac{\theta}{1-\theta} \mathbf{e}\right) \mathrm{d} s .
\end{aligned}
$$

The definition of the function $F^{*}$ may be used to replace the integrand in the second integral by $\left[\theta^{2} /(1-\theta)^{2}\right] F^{*}(e, \mathbf{e})$. If we now let $\Delta \rightarrow 0^{+}$(i.e. $\left.\Delta_{1}, \Delta_{2} \rightarrow 0^{+}\right)$with $\theta$ fixed, and invoke the mean-value theorem, we obtain

$$
\Delta^{-1} F \rightarrow \frac{\theta}{1-\theta} F^{*}(e, \mathbf{e})
$$

A similar argument applied to the functional $G$ yields

$$
\Delta^{-1} G=\frac{\theta}{\Delta_{1}} \int_{s_{1}}^{s_{2}} G^{*}(e, \mathbf{a}, \mathbf{e}) \mathrm{d} s+\frac{1-\theta}{\Delta_{2}} \int_{s_{2}}^{s_{3}} G^{*}\left(-\frac{\theta}{1-\theta} e, \mathbf{a},-\frac{\theta}{1-\theta} \mathbf{e}\right) \mathrm{d} s .
$$

As $\Delta \rightarrow 0^{+}$, the continuous function $f(s)$ defined by (5.13) approaches zero pointwise, while its derivatives remain finite in the intervals $\left(s_{1}, s_{2}\right)$ and $\left(s_{2}, s_{3}\right)$. Thus a $(s)$ approaches zero pointwise, and

$$
\Delta^{-1} G \rightarrow \theta G^{*}(e, \mathbf{0}, \mathbf{e})+(1-\theta) G^{*}\left(-\frac{\theta}{1-\theta} e, \mathbf{0},-\frac{\theta}{1-\theta} \mathbf{e}\right) .
$$

But $G^{*}(\cdot, \mathbf{0}, \cdot)=0$ by $(5.10)_{2}$, so $\Delta^{-1} G \rightarrow 0$. Thus we have shown that

$$
F=O(\Delta), \quad G=o(\Delta) ; \quad \Delta \rightarrow 0^{+}
$$

for the particular variations defined by (5.12-5.14) and (5.16).

Substitution of (5.18) and (5.21) into (5.15) yields

$$
\Delta\left[\frac{\theta}{1-\theta} F^{*}(e, \mathbf{e})+\Delta^{-1} o(\Delta)\right] \geqslant 0
$$


Dividing by $\Delta$, passing to the limit, and recalling that $0<\theta<1$, we obtain the Legendre necessary condition $F^{*}(e, \mathbf{e}) \geqslant 0$ :

$$
\left(\partial^{2} w / \partial \lambda^{2}\right) e^{2}+\mathbf{e} \cdot \mathbf{C e}+2 e \mathbf{D} \cdot \mathbf{e} \geqslant 0 \quad \text { for all } e, \mathbf{e}
$$

This holds at every point $s \in[0, L]$ in a minimizing configuration. Obvious necessary conditions for this are

$$
\partial^{2} w / \partial \lambda^{2} \geqslant 0 \text { and } \mathbf{e} \cdot \mathbf{C e} \geqslant 0, \forall \mathbf{e}
$$

The first requires that the extensional modulus be nonnegative, while the second states that the tensor of moduli associated with torsion and flexure is nonnegative definite (see (3.5), (3.23)). The latter result is equivalent to the Legendre necessary condition for inextensible rods [3].

In general, (5.23) is equivalent to the nonnegative definiteness of the $4 \times 4$ matrix

$$
\left(\begin{array}{ll}
\partial^{2} w / \partial \lambda^{2} & D_{j} \\
D_{i} & C_{i \jmath}
\end{array}\right)
$$

Necessary and sufficient conditions in terms of the elements of the matrix may be derived with the aid of a theorem for semi-definite quadratic forms given in [9, Chapt. 10].

Finally, we remark that our restriction to nodal connections of the ball and socket type is not essential. Inequality (5.23) may be shown to be necessary for all node types.

(c) Cable networks

It is of interest to re-examine the foregoing analysis for the case of a system of elastic cables. These may be modelled by suppressing the dependence of the strain energy on the variables $\kappa_{\imath}$. In this case the appropriate nodal conditions are precisely those that we have imposed previously to illustrate the theory of the second variation. Retracing the argument in the present case, we find that (5.15) is replaced by the inequality $H \geqslant 0$, where

$$
H=\int_{0}^{L} H^{*}(a, \mathbf{a}) \mathrm{d} s
$$

and

$$
\begin{aligned}
H^{*}(a, \mathbf{a}) & =F^{*}(a, \mathbf{0})+G^{*}(0, \mathbf{a}, \mathbf{0}) \\
& =(\mathrm{d} f / \mathrm{d} \lambda) a^{2}-f(\lambda) \mathbf{t} \cdot \mathbf{a} \times\left(\mathbf{a} \times \mathbf{r}^{\prime}\right) .
\end{aligned}
$$

Here

$$
f(\lambda)=\mathrm{d} w(\lambda) / \mathrm{d} \lambda
$$

and we have used the result

$$
\mathbf{F}=f(\lambda) \mathbf{t}
$$

to eliminate the second term in the expression $(5.10)_{2}$ for the function $G^{*}$. 
The absence of $\mathbf{a}^{\prime}(s)$ in (5.26) allows us to consider variations $\mathbf{a}(s)$ having less continuity than would otherwise be required. In particular, replacement of $\mathbf{a}(s)$ in $(5.12)_{1}$ with $\mathbf{a}=\mathbf{e} f^{\prime}(s)$ leads to the limit

$$
\Delta^{-1} H \rightarrow \frac{\theta}{1-\theta} H^{*}(e, \mathbf{e}) ; \quad \Delta \rightarrow 0^{+},
$$

and (5.23) is replaced by the Legendre condition for cables:

$$
H^{*}(e, \mathbf{e}) \geqslant 0 \text { for all } e, \mathbf{e} \text {. }
$$

Next, we use the identity

$$
\mathbf{F} \cdot \mathbf{e} \times\left(\mathbf{e} \times \mathbf{r}^{\prime}\right)=(\mathbf{e} \cdot \mathbf{F})\left(\mathbf{e} \cdot \mathbf{r}^{\prime}\right)-|\mathbf{e}|^{2} \mathbf{F} \cdot \mathbf{r}^{\prime},
$$

together with (5.26) and (5.28), to write (5.30) in the form

$$
(\mathrm{d} f / \mathrm{d} \lambda) e^{2}+\lambda f\left[|\mathbf{e}|^{2}-(\mathbf{t} \cdot \mathbf{e})^{2}\right] \geqslant 0 \text { for all } e, \mathbf{e} .
$$

The choice $\mathbf{e}=\mathbf{0}$ yields the counterpart of $(5.24)_{1}$ :

$$
\mathrm{d} f / \mathrm{d} \lambda \geqslant 0 .
$$

Since the bracketed term in (5.32) is nonnegative and may be positive, the alternative choice $e=0$ gives

$$
f(\lambda) \geqslant 0 \text {. }
$$

Thus, according to the idealized theory used here, the cable cannot support a compressive force in stable equilibrium. This restriction has no counterpart in the Legendre condition for rods.

Conversely, inequalities (5.33), (5.34) imply (5.32), which is equivalent to (5.30). This in turn implies that $H \geqslant 0$ for each cable, which guarantees the nonnegativity of the second variation for the entire collection of cables. The pointwise conditions (5.33), (5.34) are therefore necessary and sufficient for the local stability, in the sense of the second variation, of an elastic cable network. For rods, the relevant Legendre inequality is generally not sufficient for stability.

\section{Acknowledgement}

I gratefully acknowledge the support of the Natural Sciences and Engineering Research Council of Canada through grant OGP 0041743.

\section{References}

1. Antman, S.S., Nonlinear Problems of Elasticity, Springer-Verlag, New York, 1995.

2. Ewing, G.M., Calculus of Variations with Applications, Norton, W.W., New York, 1969.

3. Steigmann, D.J. and Faulkner, M.G., 'Variational theory for spatial rods', J. Elast., 33 (1993) 1-26.

4. Love, A.E.H., A Treatise on the Mathematical Theory of Elasticity, Cambridge University Press, 1927.

5. Dill, E.H., 'Kirchhoff's theory of rods', Archive for History of Exact Sciences, 44 (1992) 1-23.

6. Cannarozzi, M., 'Stationary and extremum variational formulations for the elastostatics of cable networks', Meccanica, 20 (1985) 136-143.

7. Tadjbakhsh, I.G. and Lagoudas, D.C., 'Variational theory of motion of curved, twisted and extensible elastic rods', Int. J. Engng. Sci., 32 (1994) 569-577.

8. Friedman, A., Partial Differential Equations, Holt, New York, 1969.

9. F.R. Gantmacher, The Theory of Matrices, Vol. 1. Chelsea, New York, 1977. 